\title{
A Great Concern Regarding the Authenticity Identification and Quality Control of Chinese Propolis and Brazilian Green Propolis
}

\author{
Shengwei Sun, Jian He, Meijuan Liu, Guangling Yin, Xuguang Zhang* \\ Science and Technology Centre, By-Health Co. Ltd, No. 3 Kehui 3rd Street, \\ No. 99 Kexue Avenue Central, Science City, Luogang District, Guangzhou 510000, China \\ *Corresponding author: zhangxg2@by-health.com
}

Received September 10, 2019; Revised October 15, 2019; Accepted October 23, 2019

\begin{abstract}
Propolis (bee glue) is a natural resin mixture produced by honey bees and it is composed of substances collected from parts of plants, buds and exudates. The composition of propolis varies primary on the geographical locations of the ingredients obtained by the bees. A series of biological properties of propolis including antimicrobial, immunostimulatory, anti-inflammatory, anti-tumor and anti-oxidation activities have been reported in relation to their chemical composition, plant origin and geographical area. This review collates the scientific literature regarding Chinese propolis and Brazilian green propolis, specifically focusing on their different constituents and biological activities. While there are known differences in composition of these two kinds of propolis, they share considerable similarity in their overall chemical composition. Importantly, techniques such as principal component analysis (PCA) and fingerprint are viable options for authenticity identification and quality control of propolis. Many of the molecular mechanisms driving various biological activities of propolis have been determined. We have also found that the biological activity of propolis mainly depends on its constituents, such as flavonoids or phenolic compounds. Each activity is regulated by a variety of biological pathways. The compounds in both Chinese propolis and Brazilian green propolis were found to participate in different biological pathways through distinct molecular mechanisms due to their diverse chemical compositions and unique structures. With the growing market interest of Brazilian green propolis in China, an evaluation between the chemical diversity and biological activity of Chinese propolis and Brazilian green propolis are in need of a review.
\end{abstract}

Keywords: Chinese propolis, Brazilian green propolis, chemical diversity, biological activity

Cite This Article: Shengwei Sun, Jian He, Meijuan Liu, Guangling Yin, and Xuguang Zhang, "A Great Concern Regarding the Authenticity Identification and Quality Control of Chinese Propolis and Brazilian Green Propolis.” Journal of Food and Nutrition Research, vol. 7, no. 10 (2019): 725-735. doi: 10.12691/jfnr-7-10-6.

\section{Introduction}

Propolis is a mixture of resin and gum collected by honeybees from the bark and bud of some plants species, such as populous and Baccharis dracunculifolia. Numerous scientific articles researching the chemical composition and biological activity of propolis have been published within the past years in different international journals by several research groups. Even at a clinical level, propolis was also observed as a useful treatment for patients with Type 2 diabetes mellitus [1]. Raw propolis is typically composed of $30 \%$ waxes, $50 \%$ plant resins, $10 \%$ essential oils, 5\% pollen and other substances [2]. As research progresses, the number of distinct natural compounds identified in propolis continues to increase.

In China and other temperate zones in Asia, the poplar trees are the main plant source of propolis [3,4]. Propolis in these regions shares similar chemical compositions including phenolic acids, flavonoids and their esters [5].
The predominant bioactive constituents presented in poplar tree buds and poplar type propolis, such as galangin, kaempferol, chrysin, pinocembrin, quercetin and some phenolic acids have been reported [6]. In addition, a series of studies have demonstrated that Chinese propolis (poplar type) has strong anti-diabetic, anti-inflammatory, antioxidant activities [7-11], which reflects the diversity and abundance of the flavonoids present in Chinese propolis. The chemical composition of Brazilian green propolis derived from Baccharis dracunculifolia DC and their parent plant were investigated and the results revealed they have similar chemical composition. Reasons may be that both of the two Brazilian strains contain a great amount of prenylated $p$-Coumaric acids including baccharin and artepillin C. Comparative studies found that propolis exhibits a series of biological activities including immunomodulatory [12], anti-inflammatory effects [13,14,15], anti-ulcer [16], antimicrobial [17], antioxidant [18], antileishmanial [19], antimutagenic [20].

The slight difference in biological properties between the two kinds of propolis mainly results from the 
difference in their chemical composition, which is because in different geographical origins, bees collect propolis from different plants in different ecosystems. Exploration of the major active compounds in propolis from different countries using different analytical methods is completely necessary. Moreover, the composition and botanical sources of propolis should be investigated in order to understand how these factors influence the biological properties of propolis [3,21].

In 2018, Brazilian green propolis was included in China's national food safety standards, which means that Brazilian green propolis will enter the Chinese market drastically and poses an unpredictable competition to Chinese propolis. Propolis has been used for different purposes and a great number of propolis-containing products have been marketed, however, confusion remains about the authenticity identification. An overview of the different methods applied to the study of Chinese propolis and Brazilian green propolis, with emphasis on authenticity identification, is presented herein. Despite the increasing use of propolis worldwide, only a few studies have been performed on the biological activities and molecular mechanisms related to the beneficial effects of the propolis components. Collectively, this review is the first focusing on the exhibition between Chinese propolis and Brazilian green propolis and the molecular mechanisms driving their biological activities.

\section{Chemical Diversity}

Geographical location is the main reason for the different chemical compositions of propolis. At present, there are mainly four kinds of propolis recognized worldwide: European, Brazilian, Cuban, and Taiwanese (Chinese) [22]. Botanical origins and pharmacological reports of propolis fundamentally have a close relationship with extensive variation in chemical constituents of propolis $[2,21,23]$. The chemical diversity of Chinese propolis and Brazilian green propolis is discussed in this review (see Table 1 ).

\subsection{Chemical Composition}

More than 300 chemical constituents have been found in propolis obtained from different regions, such as terpenoids, steroids, polyphenols (flavonoids, phenolic acids, and their esters) and amino acids [24]. A study investigating the chemical composition and antioxidant activity of the water extract of propolis (WEP) harvested from 26 different places in China showed that the major functional phenolic compounds in Chinese WEP include epicatechin, naringenin, morin, chrysin, pinocembrin, p-coumaric acid, 3,4-dimethoxycinnamic acid, ferulic acid and cinnamic acid [25].

The constituents in ethanol extracts of propolis (EEP) obtained from the following provinces of China were identified and quantitatively analyzed: Hebei, Heilongjiang, Neimenggu, Shandong, Shanxi, Henan, Gansu, Sichuan, Hainan, Hunan, Hubei and Yunnan. The results showed that the propolis exhibiting strong antioxidant activities usually contains a great number of antioxidant compounds, such as pinobanksin, rutin, quercetin, chrysin, pinobanksin 5-methylether, caffeic acid, ferulic acid, 3,4-dimethoxycinnamic acid, cinnamylideneacetic acid, p-coumaric acid, caffeic acid phenethylester, pinocembrin, apigenin, baicalein, tectochrysin, pinobanksin 3-acetate, galangin, and cinnamyl caffeate [26,27].

In Brazil, propolis was classified to 12 different kinds according to the plant source and biological characteristics of propolis. Chemical evidences suggest that the main botanical origin for Brazilian green propolis is Baccharis dracunculifolia, which locates in southern region of Brazil $[28,29]$. The predominant constituents in aqueous extract of Brazilian green propolis including cinnamic acid derivatives (p-coumaric acid, artepillin C, drupanin, and baccharin) and caffeoylquinic acid derivatives (3,4-di-O-caffeoylquinic acid, 3,5-di-O-caffeoylquinic acid, and chlorogenic acid) were evaluated for neuroprotective effects against retinal damage [30,31].

Flavonoids, phenolic acids, and phenolic acid derivative identified with different analyses are the main chemical constituents in EEP. Characterization of the phenolic compounds in Brazilian green propolis ethanol extract was surveyed using liquid chromatography-electrospray ionization mass spectrometry (LC-ESI/MS) based on the compounds' spectral characteristics. The samples contained ferulic acid, caffeoylquinic acid isomer, p-coumaric acid, caffeic acid, coumaric acid prenyl ester, dicaffeoylquinic acid isomer, caffeic acid derivative, dimethyl-dicaffeoylquinic acid, tricaffeoylquinci acid, kempferol, narigenin, isorhamnetin, hesperitin, izosakuranetin, sakuranetin, drupanin, pinocembrin, kaempferide, artepillin C derivative and kaempferide derivative [32]. The flavonoids' quantity was usually applied as the criterion to assess the quality of temperate propolis [33]. From 2000 to 2012, 112 flavonoids have been identified from different types of propolis. Dietary flavonoids, including quercetin, apigenin and baicalein are the main flavonoids in EEP, which have been shown to exert strong biological activity such as anti-oxidation [34].

Despite the differences in composition, different propolis share a lot of similarities in their chemical properties. There are many similar constituents existing both in Chinese propolis and Brazilian green propolis, such as p-coumaric acid, ferulic acid (phenolic acid), caffeic acid, apigenin, baicalein, quercetin (flavonoid) and pinocembrin. However, the unique rutin ingredient of Chinese propolis was not detected in Brazilian green propolis. Conversely, the artepillin $\mathrm{C}$ in Brazilian green propolis is a unique characteristic active substance not contained in Chinese propolis. The crude propolis collected by bee factories cannot be directly used, and it must be extracted by solvents, purified and processed before it can be used, during which the impurity components are removed and polyphenolic fractions are preserved, which is deemed to contribute more to the chemical diversity than the different geographic origins. Moreover, the analysis of propolis polyphenols by different methods results in significant differences in amount of these active compounds. 
Table 1. A comparison of chemical composition of Chinese propolis and Brazilian green propolis

\begin{tabular}{|c|c|c|c|c|}
\hline Compounds & $\mathrm{CP}$ & $\mathrm{BP}$ & Analytical techniques & references \\
\hline Epicatechin & + & $\mathrm{U}$ & RP-HPLC-DAD & {$[25]$} \\
\hline naringenin & + & $\mathrm{U}$ & RP-HPLC-DAD & {$[25]$} \\
\hline Morin & + & $\mathrm{U}$ & RP-HPLC-DAD & {$[25]$} \\
\hline chrysin & + & $\mathrm{U}$ & RP-HPLC-DAD/ HPLC-UV & {$[25]$} \\
\hline pinocembrin & + & + & RP-HPLC-DAD/ HPLC-UV & {$[25,27]$} \\
\hline$p$-coumaric acid & + & + & HPLC-ESI/MS & {$[25,46]$} \\
\hline 3,4-dimethoxycinnamic acid & + & & RP-HPLC-DAD & [25] \\
\hline Ferulic acid & + & + & RP-HPLC-DAD/ HPLC-PDA-MS & {$[25,26]$} \\
\hline Cinnamic acid & + & + & RP-HPLC-DAD & [25] \\
\hline Pinobanksin & + & $\mathrm{U}$ & HPLC-PDA-MS & {$[26]$} \\
\hline Rutin & + & - & HPLC-UV & {$[27]$} \\
\hline quercetin & + & + & HPLC-UV & {$[27]$} \\
\hline Pinobanksin 5-methylether & + & $\mathrm{U}$ & HPLC-PDA-MS & {$[26]$} \\
\hline Caffeic acid & + & $\mathrm{U}$ & HPLC-PDA-MS & {$[26]$} \\
\hline Caffeic acid phenethyl ester (CAPE) & + & $\mathrm{U}$ & HPLC-PDA-MS & {$[26]$} \\
\hline Apigenin & + & + & HPLC-UV & {$[27]$} \\
\hline Baicalein & + & + & HPLC-PDA-MS & [26] \\
\hline tectochrysin & + & $\mathrm{U}$ & HPLC-PDA-MS & {$[26,27]$} \\
\hline Pinobanksin 3-acetate & + & $\mathrm{U}$ & HPLC-PDA-MS & {$[26,27]$} \\
\hline Galangin & + & $\mathrm{U}$ & HPLC-UV & [27] \\
\hline Cinnamyl caffeate & + & $\mathrm{U}$ & HPLC-UV & {$[26,27]$} \\
\hline Artepillin C & - & + & HPLC-ESI-MS & [30] \\
\hline baccharin & $\mathrm{U}$ & + & HPLC-UV & {$[27]$} \\
\hline Caffeoylquinic acid derivatives & $\mathrm{U}$ & + & HPLC-ESI-MS & {$[30,31]$} \\
\hline kempferol & $\mathrm{U}$ & + & LC-ESI/MS/ HPLC-UV & {$[27,32]$} \\
\hline Narigenin & $\mathrm{U}$ & + & LC-ESI/MS & [32] \\
\hline Isorhamnetin & $\mathrm{U}$ & + & LC-ESI/MS & {$[32]$} \\
\hline Sakuranetin & $\mathrm{U}$ & + & LC-ESI/MS & {$[32]$} \\
\hline Drupanin & $\mathrm{U}$ & + & LC-ESI/MS & {$[32]$} \\
\hline Kaempferide & $\mathrm{U}$ & + & LC-ESI/MS & {$[32]$} \\
\hline
\end{tabular}

Note: CP: Chinese propolis; BP: Brazilian green propolis; +: detected; -: not detected; U: unconfirmed.

Table 2. A comparison of different analytical methods

\begin{tabular}{lll}
\hline & Advantages & Disadvantages \\
\hline TLC & Rapidity, simplicity, high operability & Inaccurate, only be qualitative \\
HPLC & High reliability, high recognition & Additional standard to quantify \\
CE & Novelty, rapidity, high efficiency & Poor repeatability \\
GC-MS & Good resolution, high efficiency & Additional derivatization \\
HPLC-MS & Rapidity, High reliability, high recognition, strong repeatability & Optimized method \\
\hline
\end{tabular}

\subsection{Analytical Method to Identify the Chemical Constituents}

A suitable analytical method used for the conventional examination of chemical constituents in propolis is beneficial to understanding the biological activities and enhancing the product quality control (see Table 2). A series of methods have been developed and applied to analyze propolis including thin layer chromatography (TLC) [28], spectrophotometry [35,36], high performance liquid chromatography (HPLC) [37,38], capillary electrophoresis (CE) [39,40], gas chromatography-mass spectrometry (GC-MS) [41] and high performance liquid chromatography-mass spectrometry (HPLC-MS) [42] have been specifically developed to analyze propolis. Spectrophotometry, which is based on the chromogenic reaction, has been frequently used to determine the content of flavonoids in propolis. TLC has been used to rapidly screen propolis for bioactive components prior to more detailed instrumental analysis. Despite GC can provide good resolution, it usually requires additional derivatization step before analysis. CE has been considered as a relatively novel separation technology with powerful advantages of low cost, rapidity and high separation efficiency [43], and can be an alternative to HPLC for compounds analysis. However, the repeatability of CE is inferior to the HPLC method. Among these methods, HPLC is still the most reliable and prevalent analytical tool for the characterizing polyphenol compounds in propolis [44,45].

The major compounds and content of $p$-coumaric acid in propolis recovered from the Changbai Mountains area (CBM) propolis were identified by HPLC-ESI/MS and HPLC-UV. This study also found the content of an unknown compound in CBM propolis was higher than in propolis from other places of China [46]. Mok-Ryeon Ahn 
(2007) identified the main components in EEP using HPLC combined with a photodiode array (PDA) and MS, and quantitative analysis was performed for each constituent [26]. Moreover, ethanol and dichloromethane extracts of a batch of Brazilian green propolis derived from Baccharis dracunculifolia were analyzed by high-performance liquid chromatography-atmospheric pressure chemical ionization tandem mass-spectrometry (HPLC-APCI-MS) with a positive mode, which provided a complete and unequivocal chemical composition of the Brazilian green propolis samples [47]. In another recent study, the phenolic compounds in Brazilian green propolis were qualitatively and quantitatively determined using high-performance liquid chromatographic method with diode-array detection (HPLC-DAD) and ultra-performance liquid chromatography quadrupole time-of-flight mass spectrometry (UPLC-Q-TOF-MS) methods. Qualitative analysis results obtained by liquid chromatographyelectrospray ionization mass spectrometry (LC-ESI/MS) method and quantitative analysis data obtained by HPLC (using DAD detection for quantitative analysis) were presented. The results indicated that a total of 43 phenolic ingredients were discovered in the tested propolis samples and among them, 34 compounds were identified by comparing their UV and MS/MS spectra with standards or literature data [32].

In conclusion, it can be corroborated that the HPLC method combined with MS is most commonly used analytical tool to accurately and efficiently detect and identify the active substances in propolis including Chinese propolis and Brazilian green propolis.

\subsection{Authenticity Identification and Quality Control}

Propolis has emerged as one of the most popular healthy foods and is commercially available around the world [48]. The yield of propolis may vary considerably due to a variety of factors, such as climate, season, bee species, and the arduous production process involved in the artificial harvesting and extraction of raw propolis [49]. The imbalance between the high demand in the market and limited capacity of propolis production has resulted in serious adulteration of products.

Propolis is resinous and contains a complex mixture of substances with a color varying from greenish-yellow to dark brown. Chinese propolis is mainly constituted of the buds of poplar trees, giving it a dark yellow color, while Brazilian green propolis is greenish [50]. Additionally, poplar tree gum extracted from populous buds, exhibits a similar chemical composition, color and odor to the poplar-type propolis extract [51]. According to the market survey, the poplar tree gum has been artificially mixed into a counterfeit propolis. Its lower costs and difficulty to distinguish from the beehive propolis due to their similar appearance and composition have driven the tree gum an attractive source for adulterated propolis [52]. Even worse, the Chinese propolis and Brazilian green propolis were mixed with each other due to the better promotion of propolis products. Although the adulteration of propolis does not raise immediate health and safety issues, the adulteration with poplar tree gum is immoral and illegal due to unfair trading practices. In order to ensure the authenticity and quality of propolis, it is necessary to develop a sensitive, selective, effective and relatively simple assay to distinguish tree gum from propolis [53].

Many attempts have been made to study the differences in chemical compositions between poplar tree gum and propolis. Yan-Wen Wu (2008) concluded that two-dimensional infrared (2D IR) spectroscopy and the Fourier transform infrared(FT-IR) were considered as a valuable method, which can be applied to rapidly distinguish similar natural products, especially within the extracts of Chinese propolis and poplar buds (EPB) $[4,49]$. Shuai Huang (2014) found that catechol, which was isolated from the extracts of poplar buds, can be used as a new marker compound for determination of propolis adulterated with poplar tree gum [51]. Cui-ping Zhang (2011) reported salicin can be used as a characteristic marker of the genus populous to monitor the quality of propolis as a potential counterfeit poplar gum [52]. However, the underlying differences of these two natural products cannot be determined by a single component due to the complexity of ingredients in propolis and the lack of science referring to the knowledge of propolis biomarkers. Ideally, PCA and fingerprint have been proposed as important criteria for propolis authenticity identification and quality control.

\subsubsection{PCA}

Principal Components Analysis (PCA) is usually utilized to establish the correlation between different kinds of propolis samples [54,55]. The multivariate analysis in most methods is based on the PCA fundamentally. Technically, the PCA method can be used to reveal the variance in a suit of multivariate data containing a series of basic orthogonal variables. The original variables can represent a specific linear combination of the major components. In PCA, plotting data in the space defined by the largest PCs gives a fast means to visualize similarities or differences in the data set, thereby improving sample discrimination [56,57]. Consequently, PCA was intended to provide quality control and authenticity assessment of propolis.

Brazil's production of its premium Brazilian green propolis is famous and traded worldwide. China has the largest production amount of propolis, however, the quality of Chinese propolis product is not as good as that of Brazilian green propolis product [58]. Given the odours of these two types of propolis are different, exploring the aroma active ingredients in propolis is expected to help establish a system for assessing the quality of propolis. A previous study used GC-O-MS combined with PCA method to study the aroma active ingredients in 23 propolis samples from different regions of China. These techniques are beneficial to unravel the mystery of propolis odour and achieve propolis quality control [58].

Currently, GC/MS analysis with direct headspace has been used to extract the volatile compounds from propolis. Meanwhile, ESI-MS technique has been used to characterize the non-volatile compounds in propolis. PCA-assisted either headspace GC/MS and ESI-MS is regarded as an effective means for identifying marker compounds in the Brazilian green propolis samples [50].

PCA, as an efficient chemometrics technique, can be used to characterize the relationships among a group of 
variables. The most striking feature of PCA is that it can effectively establish the relationships existing in different characteristics of multivariate due to its powerful capability to manage and interpret large data sets. In a study by H.Cheng's (2013), GC-MS analysis and electronic nose combining with PCA were used to successfully distinguish the 12 raw propolis samples from four different geographical regions of China [59]. A specific example has been shown in Figure 1.

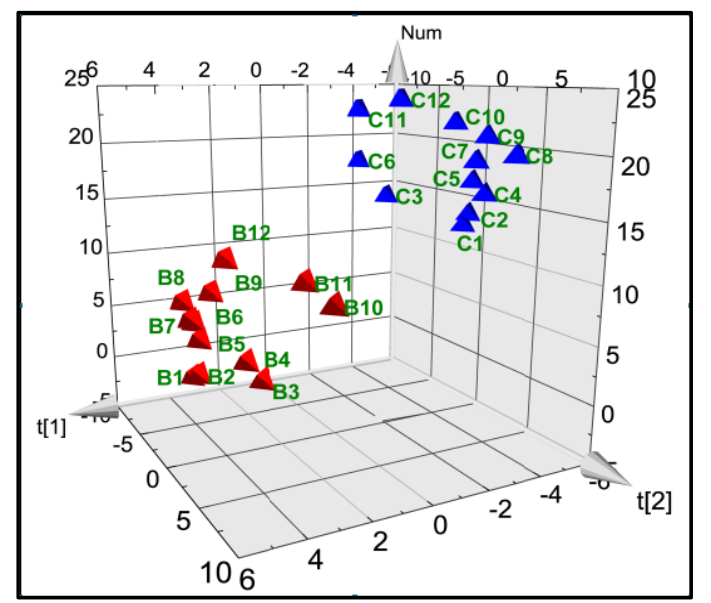

Figure 1. 3D Score plot of PCA analysis of the tested 24 batches of Brazilian and Chinese propolis HPLC fingerprints. B1-B12 represent the 12 Brazilian green propolis and C1-C12 represent the 12 Chinese propolis. The Brazilian green propolis and the Chinese propolis samples cluster into two separate categories in the score plot of PCA. (Adapted from our previous study accepted E3S Web of Conferences)

In conclusion, PCA can be strategically used in the quality control of propolis in combination with a specific analytical method. In addition, the PCA method can be used to do simultaneous on-line analysis of multiple components in propolis and theoretically solves the problem of authenticity identification of propolis, which has a broad spectrum of applications in the field of propolis research.

\subsubsection{Fingerprint}

Intensive research on the plentiful composition of propolis is being carried out with reports of new compounds annually. Therefore, it is important, particularly for Brazilian green propolis, but also for Chinese propolis, to have a direct and fast analytical procedure capable of providing reliable fingerprint characterization of crude extracts of propolis samples. Such a fingerprint would allow one to rapidly typify the propolis samples referring to authenticity identification, screen for its geographical origin, provide a reference for quality of propolis, and identify the major categories of natural product ingredients prior to initiating more characterization procedures [55].

In order to determine the plant origins of propolis samples, identification of the key characteristic marker compounds in propolis allows for a potential correlation. The fingerprint identification approach is considered an efficient and reliable method, which is suitable for analysis of a great number of samples to point out the qualitative differences among samples with complicated chemical compositions. For instance, MS fingerprint method has been successfully used for high-throughput analysis of crude propolis samples to determine the source of the samples [60]. The chemical components in different propolis samples collected from different places were investigated, and a specific fingerprint had been generated, which turned out to be useful for the quality control of extracts in pharmaceutical preparations [61]. An on-line HPLC-ESI/MS analysis can provide a solution to obtain typical propolis fingerprints and a reliable identification of a large number of polyphenolic components in propolis [62]. Specifically, if there is an unusual component in the chromatogram, the fingerprint will allow for its immediate recognition, which is beneficial for the authenticity identification of propolis.

Recently, a comparative study on the chemical profile of the Brazilian green propolis ethanoic extract was carried out between small and large apiaries. The result of ESI (-)-MS fingerprint analysis indicated that the small and large apiaries have a similar chemical profile. Moreover, the fingerprint determined by ESI (-)-MS method showed the ionization intensity of each ion. The fingerprint was automatically normalized to the most intense ion thus although different samples were assessed, the ionization intensity was compared within the same fingerprint [63]. In summary, the ESI (-)-MS fingerprint characterizes each ion, thereby facilitating the differentiation of different propolis samples. Consequently, this technique generates powerful data that can support the analysis of propolis quality.
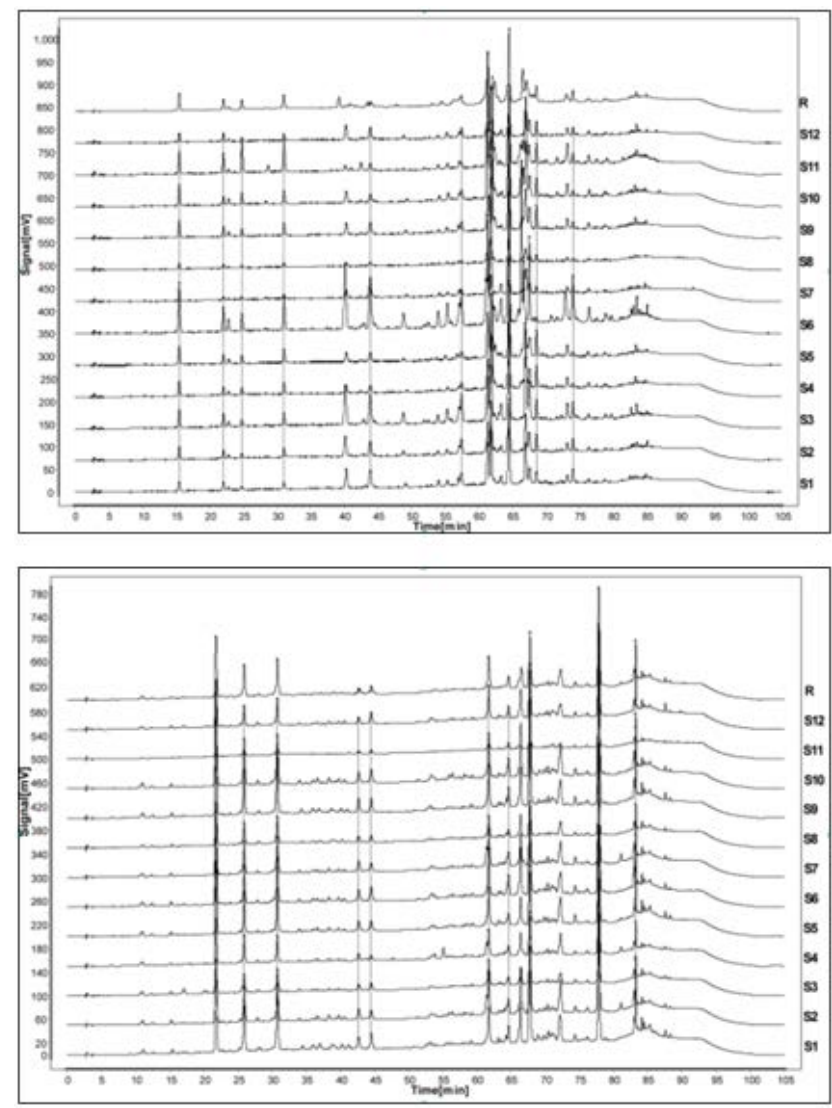

Figure 2. HPLC fingerprints of 12 batches of Brazilian green propolis (up) and Chinese propolis (down). The results demonstrate that the internal difference between the two groups of propolis samples was smaller than the external difference, indicating that the original location had a great influence on the chemical composition of propolis. (Adapted from our previous study accepted E3S Web of Conferences) 
Another example of the application of fingerprint is the development of a rapid TLC identification method, which uses chemometric fingerprint to distinguish Chinese propolis from poplar tree gum. A combination of hydrogen peroxide and ammonia vapours is used as the visualization reagent in this TLC method, which has been optimized to characterize the chemical profile of Chinese propolis. The results demonstrate the success of a proposed TLC identification strategy of Chinese propolis using chemometric fingerprint, which provides accurate sample classification $[64,65]$. A specific example of this technique is illustrated in Figure 2.

In conclusion, these results suggest that fingerprint analysis of propolis on authenticity identification and quality control is beneficial. Different types of propolis within a group of unknown samples can be identified easily and effectively, which is key application of the fingerprint through the use of a series of methods including HPLC, ESI (-)-MS and TLC. Importantly, fingerprint can provide efficient solutions to discriminate tree gum from propolis, which means it is useful for evaluating the authenticity and quality of propolis.

\section{Biological Activity and Potential Mechanism}

In the last decades, chemical composition and biological properties of propolis have become the subject of several studies extensively carried out all over the world $[66,67,68]$. In recent propolis research, the most interesting trend remains the evaluation of biological properties of propolis with different chemical compositions obtained from different geographic locations. For instance, Kujumgiev et al. compared the antimicrobial, including antibacterial, antiviral, and antifungal properties of propolis with different chemical compositions from different geographic origins. The results demonstrated that all propolis samples showed similar significant antimicrobial activity, although the chemical compositions of them varied greatly [69]. However, the specific molecular mechanisms driving the observed biological activities induced by propolis are likely different given the varying chemical composition of propolis from different geographic origins. For this reason, it is important for academic research groups to focus on elucidating the molecular mechanisms governing propolis bioactivity. Previous work has already proposed numerous mechanisms underlying the biological activity of propolis and the therapeutic applications of propolis have also been assessed, showing the importance of this bee product [70]. The combination of compounds with different chemical structures and different mechanisms of action are important for the biological activity of propolis.

This review explores the differences in the bioactivity of Chinese propolis and Brazilian green propolis as well as the differences in the underlying biological mechanisms of propolis.

\subsection{Antimicrobial Activity}

Propolis has a significant bacteriostatic effect against Enterococcus spp., Escherichia coli and Staphylococcus aureus [71,72]. Reports have also shown that ethanolic extracts of Brazilian green propolis were more effective against gram-positive bacteria while showed limited effects on gram-negative bacteria mainly owing to the abundance in flavonoids [73]. Ethanolic extracts of both Chinese propolis and Brazilian green propolis containing high contents of galangin and pinocembrin, have been proven to significantly inhibit the glucosyltransferase activity and the growth of S.mutans, implying that they may be used to prevent dental caries and oral diseases. Ntondo B. Takaisi-Kikuni (1994) reported that propolis can inhibit growth of bacterial by inhibiting cell division, and thereby inducing the formation of pseudomulticellular streptococci. Moreover, propolis can disrupt the organization of cell wall, cytoplasmic membrane and cytoplasm induced by a partial bacteriolysis, and can also inhibit protein synthesis [74]. High concentration of propolis extracts can significantly inhibit Salmonella growth, implicating the potential application of propolis in therapy of Salmonella infection [75].

Furthermore, studies have demonstrated that both ethanolic and aqueous extracts of propolis exhibit a strong antiviral activity. The ethanolic extract of propolis contains a higher amount of flavonoids, and a lower content of phenylcarboxylic acid compared to the aqueous extract [76]. In a study, both ethanolic and aqueous extracts of propolis exhibited strong antiviral activity against herpes simplex virus $\mathrm{HSV}-1$ and $\mathrm{HSV}-2$ viruses [77]. To explore the antiviral activity and mechanism of propolis extracts, cells were treated with propolis extracts before or after viral infection. The results indicated that both aqueous and ethanolic propolis extracts can significantly suppress the multiplication of HSV-2. Collectively, studies confirmed that the cytotoxic and anti-herpes effects of propolis extracts on HSV and influenza virus support the antimicrobial potential of propolis [78].

\subsection{Immunomodulatory and Antitumor Activity}

Studies indicated that the immunomodulatory effect of propolis was caused by enhancing lytic activity of natural killer cells, thereby kill tumor cells [79]. Previous studies showed that water-soluble derivative(WSD) of natural propolis can stimulate macrophages and influence immune defense mechanisms via inducing macrophage phagocytosis enhancing release of migration inhibitors, and increasing the number of rosette-forming and antibody-producing cells [80,81]. Activated macrophages were major component of the host defense against tumor growth [80,81]. The elevated levels of TNF and IL-1 produced by activated macrophages are directly related to two other criteria of macrophage activation: macrophageinduced tumor cytotoxicity and enhanced response to chemotactic stimuli in vitro [82,83]. Toll-like receptor (TLR) plays a crucial role in the development of adaptive immune responses and microbial recognition. It has been reported that propolis inhibits the initial steps of the immune response in vivo by down-regulating production of pro-inflammatory cytokines and the expression of TLR4 and TLR2, suggesting that propolis can modulate the innate immunity [84]. 
Nada Oršolić (2005) reported that water-soluble derivative and polyphenolic compounds from propolis can suppress tumor growth via orally given to mice. Local presence of caffeic acid and caffeic acid phenethyl ester (CAPE) both in Chinese propolis and Brazilian green propolis significantly suppressed tumor growth. It was postulated that the antitumor activity of propolis was casued by imunomodulatory activity, including the enhancement of non-specific antitumor effect via macrophage activation and the production of soluble factors which can influence either immune cells or directly by tumor cells [85]. Importantly, the primary active compound CAPE found in propolis is considered to be the main contributor of a series of biological properties, including its antitumor activity. It has been demonstrated that CAPE can cause acetylated histones accumulation in MDA-MB-231 (ER-/PR-/Her2-) and MCF-7 (ER+) cells, which is associated with up-regulated ER and downrelulated EGFR in MDA-MB-231 cells and decreased ER and PR in MCF-7 cells. This evidence provides a potential mechanistic basis for propolis activity in breast cancer, which can be optimized for therapeutic intervention in cancer [86,87]. In addition, the results of in vivo and in vitro studies suggest that chrysin in both Chinese propolis and Brazilian green propolis may inhibit tumor cell progression and may be useful as a protential chemotherapeutic or chemopreventive antitumor reagent [88].

\subsection{Anti-inflammatory Activity}

Many studies have shown that propolis has significant anti-inflammatory effect on both chronic and acute inflammation [89]. Some substances in propolis have the capacity to inhibit cyclooxygenase and the consequent synthesis of prostaglandins [90]. This is considered to be part of the anti-inflammatory mechanisms of propolis. Furthermore, the molecular compounds exerting antioxidant and lipoxygenase (LOX) inhibitory activities have potential anti-inflammatory activity as well. Studies have reported the anti-inflammatory activities of propolis and its sub-products in different models of both acute and chronic inflammation. In many of these studies, the anti-inflammatory activity of propolis was observed to be similarly strong to that of the anti-inflammatory drugs, which were used as positive controls in the experiments.

Reports showed that the aqueous propolis extracts exhibited specific effects including inhibition of 5-LOX activity, prostaglandin biosynthesis and platelet aggregation [91]. Moreover, propolis exhibited free radical scavenging activity in vitro and a hepatoprotective effect against cell death induced by TNF- $\alpha$ [35]. Inhibition of TNF- $\alpha$-mediated NF- $\kappa B$ activation may be the molecular basis for the anti-inflammatory properties of propolis. The propolis ethanol extract exhibited a dose-dependent anti-inflammatory effects in foreign body-induced granuloma, carrageenan-induced paw edema and Freund's adjuvant-induced arthritis models; in addition, it also affected analgesic activity and vascular permeability in mice [92].

The effect of chrysin present in propolis on the expression of cyclooxygenase-2 (COX-2) was investigated in Raw 264.7 cells activated by lipopolysaccharide (LPS) [93], and they observed that chrysin in both Chinese propolis and Brazilian green propolis significantly suppressed the expression of both COX-2 mRNA and LPS-induced COX-2 protein in a concentration-dependent manner. Electrophoretic mobility shift and mutational analyses indicated that the transcription factor for IL-6 contributes to down-regulation of chrysin-mediated COX-2, implying that this compound has antiinflammatory and anti-carcinogenic activities [93]. Artepillin C, as a characteristic compound present in Brazilian green propolis can suppress the activity of NF$\kappa \mathrm{B}$ in HEK 293 cells, decrease the production of nitric oxide in RAW 264.7 cells, and inhibit the synthesis of prostaglandin E2 in mice [15]. Another study demonstrated that the flavonoid quercetin, which exists in both Chinese propolis and Brazilian green propolis, can down-regulate inflammatory responses and support neuroprotection by reducing the production of nitric oxide and inhibiting the expression of inducible nitric oxide synthase (iNOS) in endotoxin/cytokine-stimulated microglia [94]. Besides, the quercetin showed anti-inflammatory activity by the down-regulating extracellular signal molecules including Akt, c-Jun N-terminal kinase, Janus kinase-1, Src, p38, NF- $\kappa$ B, signal transducer and activator of transcription-1, and Tyk2 [94]. Kai Wang (2012) reported that extract from Chinese propolis which is rich in flavonoids, including pinocembrin, quercetin, apigenin, kaempferol, galangin, rutin, myricetin, and chrysin, could significantly decrease the production of IL-1 $\beta$, IL- 6 and NO in LPS- stimulated RAW 264.7 cells in relation to suppress mRNA expression of IL-1 $\beta$, IL-6 and iNOS in a time- and concentration-dependent manner [95].

In summary, the anti-inflammatory properties of propolis involve many complicated pathways with distinct mechanisms in vitro and in vivo. The active compounds in Chinese propolis and Brazilian green propolis have certain anti-inflammatory activities to some extent, which has relation with the evaluation models and methods used.

\subsection{Antioxidant Activity}

Observational evidence suggests that both aqueous and ethanol extracts of propolis exert anti-oxidative effects via three different mechanisms including metal ion chelating activity, free radical scavenging activity, and hydrogen donating capability [96]. Previous work indicated that ethanol extracts of Chinese propolis enhances endogenous antioxidant defenses by directly eliminating reactive oxygen species and activating antioxidant genes (GCLM, Erk-Nrf2-HO1 and TrxR1) in RAW 264.7 cells [97]. Artepillin $\mathrm{C}$ is a phenolic compound containing isoprenyl, isolated from Brazilian green propolis and it is an effective antioxidant. It is well known that hydrogen transferring from the phenolic hydroxyl group to the active radical substances, such as superoxide anion, lipid peroxyl radical and hydroxyl radical, is the mechanism for the antioxidative activity of phenolic compounds. Recently, research reported that the hydrogen transfer rate constant from artepillin $\mathrm{C}$ to dry peroxide radical is equal to that of $(+)$ - catechin by one step hydrogen atom transfer rather than electron transfer, which indicates that artepillin C can be used as an effective antioxidant [98].

With regard to the structure and antioxidant activity of flavonoids in propolis, there have been many discussions 
and contradictions over the years. Flavonoids require 3',4'-dihydroxyl groups of the B-ring and a hydroxyl group at carbon 3 of the $\mathrm{C}$ - ring and for their antioxidant activity [99]. For example, CAPE is an active substance in propolis, decorating 3,4-dihydroxyl groups on the catechol ring which is thought to contribute to its antioxidant activity [100]. Quercetin containing 3',4'-dihydroxyl groups on the $\mathrm{B}$ - ring exhibits the most powerful antioxidant activity, kaempferol with 4'-monohydroxyl group at the B- ring shows medium antioxidant activity, and galangin without hydroxyl group at the B-ring, exhibits minimal antioxidant activity [99].

In conclusion, the antioxidant activity of propolis is currently appealing for their use in improving human health. The mechanism by which propolis exerts its antioxidant activity is also complex, involving free radicals scavenging, hydrogen ion transferring and oxidative stress reduction. Both Chinese propolis and Brazilian green propolis contain flavonoids and polyphenolic compounds with strong antioxidant activity. It is worth noting that the artepillin $\mathrm{C}$ in Brazilian green propolis has a strong ability to scavenge free radicals and therefore, has a very strong antioxidant effect.

\section{Conclusion}

Propolis research is still a new domain in natural products studies. Much work remains to be done on determining propolis chemical compositions and biological activities. Although the composition of Chinese propolis and Brazilian green propolis may differ, which provides a unique advantage in terms of their individual biological activity, the two samples share considerable similarities in their overall chemical constituents, as well. The chemical diversity in propolis is not only determined by geographical origin and for this reason more advanced and efficient analytical methods should be used to explore the composition of compounds in propolis. Additionally, products containing propolis have been widely marketed around the world. Unfortunately, adulteration is still ubiquitous in order to reduce production costs or increase the promotion of sales. To address this problem, the PCA and fingerprinting methods discussed in this review are expected to provide a reference for authenticity identification and quality control.

Propolis possesses a wide range of biological potentials including anti-microbial, immunomodulatory, anti-tumor, anti-inflammatory and anti-oxidant activities. The mechanisms of each biological activity induced by distinct chemical compounds in propolis have been investigated in this review. We found that the biological activity of propolis depends mainly on the constituents therein, such as flavonoids or phenolic compounds. However, most studies only investigated the effect of bioactive compounds in vitro using cells model and the related mechanisms including specific pathways in vivo still remain unidentified. The mechanisms underlying the beneficial effects of propolis are multifactorial and are regulated by a variety of biological pathways. The compounds in the propolis affect multiple pathways in vitro and in vivo due to the diverse chemical compositions and unique structures of propolis constituents. Therefore, the combination of different compounds each with unique chemical structures, as well as the distinct mechanisms of action of each compound are important for the various biological activities induced by propolis. In conclusion, both types of propolis have their own characteristics, and the excellent complementarity of propolis products on the market can jointly promote human health.

\section{Conflicts of Interest}

There are no conflicts to declare.

\section{References}

[1] Zakerkish, M., Jenabi, M., Zaeemzadeh, N., Hemmati, A. A., and Neisi, N. (2019) The Effect of Iranian Propolis on Glucose Metabolism, Lipid Profile, Insulin Resistance, Renal Function and Inflammatory Biomarkers in Patients with Type 2 Diabetes Mellitus: A Randomized Double-Blind Clinical Trial, Scientific reports 9 .

[2] Burdock, G. (1998) Review of the biological properties and toxicity of bee propolis (propolis), Food and Chemical toxicology 36, 347-363.

[3] Sforcin, J. M., and Bankova, V. (2011) Propolis: is there a potential for the development of new drugs?, Journal of ethnopharmacology 133, 253-260.

[4] Wu, Y.-W., Sun, S.-Q., Zhao, J., Li, Y., and Zhou, Q. (2008) Rapid discrimination of extracts of Chinese propolis and poplar buds by FT-IR and 2D IR correlation spectroscopy, Journal of Molecular Structure 883, 48-54.

[5] Toreti, V. C., Sato, H. H., Pastore, G. M., and Park, Y. K. (2013) Recent progress of propolis for its biological and chemical compositions and its botanical origin, Evidence-based complementary and alternative medicine 2013.

[6] Rubiolo, P., Casetta, C., Cagliero, C., Brevard, H., Sgorbini, B., and Bicchi, C. (2013) Populus nigra L. bud absolute: a case study for a strategy of analysis of natural complex substances, Analytical and bioanalytical chemistry 405, 1223-1235.

[7] Kai, W., Shun, P., Shuai, H., Lin, H., Hongzhuan, X., Cuiping, Z., and Fuliang, H. (2013) Molecular mechanisms underlying the in vitro anti-inflammatory effects of a flavonoid-rich ethanol extract from chinese propolis (poplar type), Evidence-Based Complementray and Alternative Medicine,2013,(2013-1-15) 2013, 127672.

[8] Krol, W., ., Czuba, Z., ., Scheller, S., ., Gabrys, J., ., Grabiec, S., ., and Shani, J., . (1990) Anti-oxidant property of ethanolic extract of propolis (EEP) as evaluated by inhibiting the chemiluminescence oxidation of luminol, Biochemistry International 21, 593.

[9] Mirzoeva, O. K., and Calder, P. C. (1996) The effect of propolis and its components on eicosanoid production during the inflammatory response, Prostaglandins Leukotrienes \& Essential Fatty Acids 55, 441

[10] Wei, Z., Ying-Hua, L., Min-Li, C., and Fu-Liang, H. (2011) Protective effects of Chinese and Brazilian propolis treatment against hepatorenal lesion in diabetic rats, Human \& Experimental Toxicology 30, 1246-1255.

[11] Xuan, H., Zhu, R., Li, Y., and Hu, F. (2010) Inhibitory effect of chinese propolis on phosphatidylcholine-specific phospholipase $\mathrm{C}$ activity in vascular endothelial cells, Evid Based Complement Alternat Med 2011, 985278.

[12] Missima, F., Filho, A. A. d. S., Nunes, G. A., Bueno, P. C. P., De Sousa, J. P. B., Bastos, J. K., and Sforcin, J. M. (2007) Effect of Baccharis dracunculifolia DC (Asteraceae) extracts and its isolated compounds on macrophage activation, Journal of Pharmacy and Pharmacology 59, 463-468.

[13] Cestari, S. H., Bastos, J. K., and Di Stasi, L. C. (2011) Intestinal anti-inflammatory activity of Baccharis dracunculifolia in the trinitrobenzenesulphonic acid model of rat colitis, Evidence-Based Complementary and Alternative Medicine 2011. 
[14] dos Santos, D. A., Fukui, M. d. J., Nanayakkara, N. D., Khan, S. I., Sousa, J. P. B., Bastos, J. K., Andrade, S. F., da Silva Filho, A. A., and Quintão, N. L. (2010) Anti-inflammatory and antinociceptive effects of Baccharis dracunculifolia DC (Asteraceae) in different experimental models, Journal of Ethnopharmacology 127, 543550.

[15] Paulino, N., Abreu, S. R. L., Uto, Y., Koyama, D., Nagasawa, H., Hori, H., Dirsch, V. M., Vollmar, A. M., Scremin, A., and Bretz, W. A. (2008) Anti-inflammatory effects of a bioavailable compound, Artepillin C, in Brazilian propolis, European Journal of Pharmacology 587, 296-301.

[16] Lemos, M., De Barros, M. P., Sousa, J. P. B., Filho, A. A. d. S., Bastos, J. K., and De Andrade, S. F. (2007) Baccharis dracunculifolia, the main botanical source of Brazilian green propolis, displays antiulcer activity, Journal of Pharmacy and Pharmacology 59, 603-608.

[17] da Silva Filho, A. A., de Sousa, J. P., Soares, S., Furtado, N. A., e Silva, M. L. A., Cunha, W. R., Gregório, L. E., Nanayakkara, N. D., and Bastos, J. K. (2008) Antimicrobial activity of the extract and isolated compounds from Baccharis dracunculifolia DC (Asteraceae), Zeitschrift für Naturforschung C 63, 40-46.

[18] Guimarães, N. S., Mello, J. C., Paiva, J. S., Bueno, P. C., Berretta, A. A., Torquato, R. J., Nantes, I. L., and Rodrigues, T. (2012) Baccharis dracunculifolia, the main source of green propolis, exhibits potent antioxidant activity and prevents oxidative mitochondrial damage, Food and Chemical Toxicology 50, 10911097.

[19] da Silva Filho, A., Resende, D., Fukui, M., Santos, F., Pauletti, P., Cunha, W., Silva, M., Gregório, L., Bastos, J., and Nanayakkara, N. (2009) In vitro antileishmanial, antiplasmodial and cytotoxic activities of phenolics and triterpenoids from Baccharis dracunculifolia DC (Asteraceae), Fitoterapia 80, 478-482.

[20] Munari, C. C., Resende, F. A., Alves, J. M., de Sousa, J. P. B., Bastos, J. K., and Tavares, D. C. (2008) Mutagenicity and antimutagenicity of Baccharis dracunculifolia extract in chromosomal aberration assays in Chinese hamster ovary cells, Planta medica 74, 1363-1367.

[21] Bankova, V. (2005) Chemical diversity of propolis and the problem of standardization, Journal of ethnopharmacology 100, 114-117.

[22] Bankova, V. (2005) Recent trends and important developments in propolis research, Evidence-based complementary and alternative medicine 2, 29-32.

[23] Banskota, A. H., Tezuka, Y., and Kadota, S. (2001) Recent progress in pharmacological research of propolis, Phytotherapy research 15, 561-571.

[24] Bankova, V. S., de Castro, S. L., and Marcucci, M. C. (2000) Propolis: recent advances in chemistry and plant origin, Apidologie 31, 3-15.

[25] Guo, X., Chen, B., Luo, L., Zhang, X., Dai, X., and Gong, S. (2011) Chemical compositions and antioxidant activities of water extracts of Chinese propolis, Journal of agricultural and food chemistry 59, 12610-12616.

[26] Ahn, M.-R., Kumazawa, S., Usui, Y., Nakamura, J., Matsuka, M., Zhu, F., and Nakayama, T. (2007) Antioxidant activity and constituents of propolis collected in various areas of China, Food Chemistry 101, 1383-1392.

[27] Zhou, J., Li, Y., Zhao, J., Xue, X., Wu, L., and Chen, F. (2008) Geographical traceability of propolis by high-performance liquidchromatography fingerprints, Food Chemistry 108, 749-759.

[28] Park, Y. K., Alencar, S. M., and Aguiar, C. L. (2002) Botanical origin and chemical composition of Brazilian propolis, Journal of Agricultural and Food Chemistry 50, 2502-2506.

[29] Szliszka, E., Zydowicz, G., Mizgala, E., and Krol, W. (2012) Artepillin C (3, 5-diprenyl-4-hydroxycinnamic acid) sensitizes LNCaP prostate cancer cells to TRAIL-induced apoptosis, International Journal of Oncology 41, 818-828.

[30] Moura, S. A. L. d., Negri, G., Salatino, A., Lima, L. D. d. C., Dourado, L. P. A., Mendes, J. B., Andrade, S. P., Ferreira, M. A. N. D., and Cara, D. C. (2011) Aqueous extract of Brazilian green propolis: primary components, evaluation of inflammation and wound healing by using subcutaneous implanted sponges, Evidence-Based Complementary and Alternative Medicine 2011.

[31] Nakajima, Y., Shimazawa, M., Mishima, S., and Hara, H. (2007) Water extract of propolis and its main constituents, caffeoylquinic acid derivatives, exert neuroprotective effects via antioxidant actions, Life sciences 80, 370-377.
[32] Szliszka, E., Kucharska, A. Z., Sokół-Łętowska, A., Mertas, A., Czuba, Z. P., and Król, W. (2013) Chemical composition and antiinflammatory effect of ethanolic extract of Brazilian green propolis on activated J774A. 1 macrophages, Evidence-Based Complementary and Alternative Medicine 2013.

[33] Cui-ping, Z., Shuai, H., Wen-ting, W., Shun, P., Xiao-ge, S., Yajing, L., and Fu-liang, H. (2014) Development of highperformance liquid chromatographic for quality and authenticity control of Chinese propolis, Journal of food science 79, C1315C1322.

[34] Szliszka, E., Zydowicz, G., Janoszka, B., Dobosz, C., KowalczykZiomek, G., and Krol, W. (2011) Ethanolic extract of Brazilian green propolis sensitizes prostate cancer cells to TRAIL-induced apoptosis, International Journal of Oncology 38, 941-953.

[35] Alencar, S., Oldoni, T., Castro, M., Cabral, I., Costa-Neto, C., Cury, J., Rosalen, P., and Ikegaki, M. (2007) Chemical composition and biological activity of a new type of Brazilian propolis: red propolis, Journal of ethnopharmacology 113, 278283.

[36] Kalogeropoulos, N., Konteles, S. J., Troullidou, E., Mourtzinos, I., and Karathanos, V. T. (2009) Chemical composition, antioxidant activity and antimicrobial properties of propolis extracts from Greece and Cyprus, Food chemistry 116, 452-461.

[37] de Funari, C. S., de Oliveira Ferro, V., and Mathor, M. B. (2007) Analysis of propolis from Baccharis dracunculifolia DC. (Compositae) and its effects on mouse fibroblasts, Journal of ethnopharmacology 111, 206-212.

[38] Lima, B., Tapia, A., Luna, L., Fabani, M. P., SchmedaHirschmann, G., Podio, N. S., Wunderlin, D. A., and Feresin, G. E (2009) Main flavonoids, DPPH activity, and metal content allow determination of the geographical origin of propolis from the province of San Juan (Argentina), Journal of agricultural and food chemistry 57, 2691-2698.

[39] Cao, Y., Wang, Y., and Yuan, Q. (2004) Analysis of flavonoids and phenolic acid in propolis by capillary electrophoresis, Chromatographia 59, 135-140.

[40] Jiang, L., Fang, G., Zhang, Y., Cao, G., and Wang, S. (2008) Analysis of flavonoids in propolis and Ginkgo biloba by micellar electrokinetic capillary chromatography, Journal of agricultural and food chemistry 56, 11571-11577.

[41] Campo Fernández, M., Cuesta-Rubio, O., Rosado Perez, A. s., Montes De Oca Porto, R., Márquez Hernández, I., Piccinelli, A. L., and Rastrelli, L. (2008) GC-MS determination of isoflavonoids in seven red Cuban propolis samples, Journal of agricultural and food chemistry 56, 9927-9932.

[42] Gardana, C., Scaglianti, M., Pietta, P., and Simonetti, P. (2007) Analysis of the polyphenolic fraction of propolis from different sources by liquid chromatography-tandem mass spectrometry, Journal of Pharmaceutical and Biomedical Analysis 45, 390-399.

[43] Lu, Y., Wu, C., and Yuan, Z. (2004) Determination of hesperetin, cinnamic acid and nicotinic acid in propolis with micellar electrokinetic capillary chromatography, Fitoterapia 75, 267-276.

[44] Pellati, F., Orlandini, G., Pinetti, D., and Benvenuti, S. (2011) HPLC-DAD and HPLC-ESI-MS/MS methods for metabolite profiling of propolis extracts, Journal of Pharmaceutical and Biomedical Analysis 55, 934-948.

[45] Sun, S., Liu, M., He, J., Li, K., Zhang, X., and Yin, G. (2019) Identification and Determination of Seven Phenolic Acids in Brazilian Green Propolis by UPLC-ESI-QTOF-MS and HPLC, Molecules 24, 1791.

[46] Jiang, X., Tian, J., Zheng, Y., Zhang, Y., Wu, Y., Zhang, C., Zheng, H., and Hu, F. (2019) A New Propolis Type from Changbai Mountains in North-east China: Chemical Composition, Botanical Origin and Biological Activity, Molecules 24, 1369.

[47] Chang, R., Piló-Veloso, D., Morais, S. A., and Nascimento, E. A (2008) Analysis of a Brazilian green propolis from Baccharis dracunculifolia by HPLC-APCI-MS and GC-MS, Revista Brasileira de Farmacognosia 18, 549-556.

[48] Castaldo, S., and Capasso, F. (2002) Propolis, an old remedy used in modern medicine, Fitoterapia 73, S1-S6.

[49] Xu, L., Yan, S.-M., Cai, C.-B., and Yu, X.-P. (2013) Untargeted detection and quantitative analysis of poplar balata (PB) in Chinese propolis by FT-NIR spectroscopy and chemometrics, Food chemistry 141, 4132-4137.

[50] Nunes, C. A., and Guerreiro, M. C. (2012) Characterization of Brazilian green propolis throughout the seasons by headspace 
GC/MS and ESI-MS, Journal of the Science of Food and Agriculture 92, 433-438.

[51] Huang, S., Zhang, C.-P., Li, G., Sun, Y.-Y., Wang, K., and Hu, F.L. (2014) Identification of catechol as a new marker for detecting propolis adulteration, Molecules 19, 10208-10217.

[52] Zhang, C.-p., Zheng, H.-q., Liu, G., and Hu, F.-l. (2011) Development and validation of HPLC method for determination of salicin in poplar buds: Application for screening of counterfeit propolis, Food Chemistry 127, 345-350.

[53] Stan, L., Mărghitaş, L. A., and Dezmirean, D. (2011) Quality criteria for propolis standardization, Scientific Papers Animal Science and Biotechnologies 44, 137-140.

[54] Sawaya, A. C., Cunha, I. B., Marcucci, M. C., de Oliveira Rodrigues, R. F., and Eberlin, M. N. (2006) Brazilian propolis of Tetragonisca angustula and Apis mellifera, Apidologie 37, 398407.

[55] Sawaya, A. C., Tomazela, D. M., Cunha, I. B., Bankova, V. S., Marcucci, M. C., Custodio, A. R., and Eberlin, M. N. (2004) Electrospray ionization mass spectrometry fingerprinting of propolis, Analyst 129, 739-744.

[56] Abdi, H., and Williams, L. J. (2010) Principal component analysis, Wiley interdisciplinary reviews: computational statistics 2, 433-459.

[57] Wold, S., Esbensen, K., and Geladi, P. (1987) Principal component analysis, Chemometrics and intelligent laboratory systems 2, 37-52.

[58] Yang, C., Luo, L., Zhang, H., Yang, X., Lv, Y., and Song, H. (2010) Common aroma-active components of propolis from 23 regions of China, Journal of the Science of Food and Agriculture 90, 1268-1282.

[59] Cheng, H., Qin, Z., Guo, X., Hu, X., and Wu, J. (2013) Geographical origin identification of propolis using GC-MS and electronic nose combined with principal component analysis, Food research international 51, 813-822.

[60] Sawaya, A. C. H. F., da Silva Cunha, I. B., Marcucci, M. C., Aidar, D. S., Silva, E. C. A., Carvalho, C. A. L., and Eberlin, M. N. (2007) Electrospray ionization mass spectrometry fingerprinting of propolis of native Brazilian stingless bees, Apidologie 38, 93-103.

[61] Sawaya, A. C., Abdelnur, P. V., Eberlin, M. N., Kumazawa, S., Ahn, M.-R., Bang, K.-S., Nagaraja, N., Bankova, V. S., and Afrouzan, H. (2010) Fingerprinting of propolis by easy ambient sonic-spray ionization mass spectrometry, Talanta 81, 100-108.

[62] Volpi, N., and Bergonzini, G. (2006) Analysis of flavonoids from propolis by on-line HPLC-electrospray mass spectrometry, Journal of Pharmaceutical and Biomedical Analysis 42, 354-361.

[63] Corrêa, W. R., López, B. G.-C., Prado, S. C. d., Cunha, I. B. d. S., Sawaya, A. C. H. F., and Salvador, M. J. (2016) ESI-MS fingerprinting of residues of green propolis, and evaluation of their antioxidant and antimicrobial activities, Journal of Apicultural Research 55, 1-7.

[64] Milojković Opsenica, D., Ristivojević, P., Trifković, J., Vovk, I., Lušić, D., and Tešić, Ž. (2016) TLC fingerprinting and pattern recognition methods in the assessment of authenticity of poplartype propolis, Journal of chromatographic science 54, 1077-1083.

[65] Tang, T. X., Guo, W. y., Xu, Y., Zhang, S. m., Xu, X. j., Wang, D. m., Zhao, Z. m., Zhu, L. p., and Yang, D. p. (2014) Thin-layer chromatographic identification of Chinese propolis using chemometric fingerprinting, Phytochemical analysis 25, 266-272.

[66] de Groot, A. C. (2013) Propolis: a review of properties, applications, chemical composition, contact allergy, and other adverse effects, Dermatitis 24, 263-282.

[67] Król, W., Bankova, V., Sforcin, J. M., Szliszka, E., Czuba, Z., and Kuropatnicki, A. K. (2013) Propolis: properties, application, and its potential, Evidence-Based Complementary and Alternative Medicine 2013.

[68] Silva-Carvalho, R., Baltazar, F., and Almeida-Aguiar, C. (2015) Propolis: a complex natural product with a plethora of biological activities that can be explored for drug development, EvidenceBased Complementary and Alternative Medicine 2015.

[69] Kujumgiev, A., Tsvetkova, I., Serkedjieva, Y., Bankova, V., Christov, R., and Popov, S. (1999) Antibacterial, antifungal and antiviral activity of propolis of different geographic origin, Journal of ethnopharmacology 64, 235-240.

[70] Sforcin, J. M. (2016) Biological properties and therapeutic applications of propolis, Phytotherapy research 30, 894-905.
[71] Kuropatnicki, A. K., Szliszka, E., and Krol, W. (2013) Historical aspects of propolis research in modern times, Evidence-Based Complementary and Alternative Medicine 2013.

[72] Noori, A., Al-Ghamdi, A., Ansari, M. J., Al-Attal, Y., and Salom, K. (2012) Synergistic effects of honey and propolis toward drug multi-resistant Staphylococcus aureus, Escherichia coli and Candida albicans isolates in single and polymicrobial cultures, International journal of medical sciences 9, 793.

[73] Ahuja, V., and Ahuja, A. (2011) Apitherapy-A sweet approach to dental diseases. Part II: Propolis, Journal of Advanced Oral Research 2, 1-8.

[74] Takaisi-Kikuni, N. B., and Schilcher, H. (1994) Electron microscopic and microcalorimetric investigations of the possible mechanism of the antibacterial action of a defined propolis provenance, Planta Medica 60, 222-227.

[75] Orsi, R., Sforcin, J., Rall, V., Funari, S., Barbosa, L., and Fernandes, J. (2005) Susceptibility profile of Salmonella against the antibacterial activity of propolis produced in two regions of Brazil, Journal of Venomous Animals and Toxins including Tropical Diseases 11, 109-116.

[76] Nolkemper, S., Reichling, J., Sensch, K. H., and Schnitzler, P. (2010) Mechanism of herpes simplex virus type 2 suppression by propolis extracts, Phytomedicine 17, 132-138.

[77] Schnitzler, P., Neuner, A., Nolkemper, S., Zundel, C., Nowack, H., Sensch, K. H., and Reichling, J. (2010) Antiviral activity and mode of action of propolis extracts and selected compounds, Phytotherapy Research 24, S20-S28.

[78] Serkedjieva, J., Manolova, N., and Bankova, V. (1992) Antiinfluenza virus effect of some propolis constituents and their analogues (esters of substituted cinnamic acids), Journal of Natural Products 55, 294-297.

[79] Sforcin, J. (2007) Propolis and the immune system: a review, Journal of ethnopharmacology 113, 1-14.

[80] Murad, J. M., Calvi, S. A., Soares, A. M. V. C., Bankova, V., and Sforcin, J. M. (2002) Effects of propolis from Brazil and Bulgaria on fungicidal activity of macrophages against Paracoccidioides brasiliensis, Journal of Ethnopharmacology 79, 331-334.

[81] Oršolić, N., and Bašić, I. (2003) Immunomodulation by watersoluble derivative of propolis: a factor of antitumor reactivity, Journal of Ethnopharmacology 84, 265-273.

[82] Dimov, V., Ivanovska, N., Bankova, V., and Popov, S. (1992) Immunomodulatory action of propolis: IV. Prophylactic activity against gram-negative infections and adjuvant effect of the watersoluble derivative, Vaccine 10, 817-823.

[83] Meltzer, M. S., Tucker, R. W., Sanford, K. K., and Leonard, E. J. (1975) Interaction of BCG-activated macrophages with neoplastic and nonneoplastic cell lines in vitro: quantitation of the cytotoxic reaction by release of tritiated thymidine from prelabeled target cells, Journal of the National Cancer Institute 54, 1177-1184.

[84] Orsatti, C., Missima, F., Pagliarone, A., Bachiega, T. F., Búfalo, M., Araújo Jr, J., and Sforcin, J. (2010) Propolis immunomodulatory action in vivo on Toll-like receptors 2 and 4 expression and on pro-inflammatory cytokines production in mice, Phytotherapy Research 24, 1141-1146.

[85] Oršolić, N., Šaranović, A. B., and Bašić, I. (2006) Direct and indirect mechanism (s) of antitumour activity of propolis and its polyphenolic compounds, Planta medica 72, 20-27.

[86] Chang, H., Wang, Y., Yin, X., Liu, X., and Xuan, H. (2017) Ethanol extract of propolis and its constituent caffeic acid phenethyl ester inhibit breast cancer cells proliferation in inflammatory microenvironment by inhibiting TLR4 signal pathway and inducing apoptosis and autophagy, BMC complementary and alternative medicine 17, 471.

[87] Omene, C., Kalac, M., Wu, J., Marchi, E., Frenkel, K., and O’Connor, O. A. (2013) Propolis and its active component, caffeic acid phenethyl ester (CAPE), modulate breast cancer therapeutic targets via an epigenetically mediated mechanism of action, Journal of cancer science \& therapy 5, 334.

[88] Sawicka, D., Car, H., Borawska, M. H., and Nikliński, J. (2012) The anticancer activity of propolis, Folia Histochemica et Cytobiologica 50, 25-37.

[89] Uzel, A., Önçağ, Ö., Çoğulu, D., and Gençay, Ö. (2005) Chemical compositions and antimicrobial activities of four different Anatolian propolis samples, Microbiological research 160, 189195. 
[90] Sigal, L. H., and Ron, Y. (1994) Immunology and inflammation. Basic mechanisms and clinical consequences, McGaw-Hill, Inc., New York, 583.

[91] Massaro, F. C., Brooks, P. R., Wallace, H. M., and Russell, F. D. (2011) Cerumen of Australian stingless bees (Tetragonula carbonaria): gas chromatography-mass spectrometry fingerprints and potential anti-inflammatory properties, Naturwissenschaften 98, 329-337.

[92] Park, E.-H., Kim, S.-H., and Park, S.-S. (1996) Anti-inflammatory activity of propolis, Archives of Pharmacal Research 19, 337-341.

[93] Woo, K. J., Jeong, Y.-J., Inoue, H., Park, J.-W., and Kwon, T. K. (2005) Chrysin suppresses lipopolysaccharide-induced cyclooxygenase-2 expression through the inhibition of nuclear factor for IL-6 (NF-IL6) DNA-binding activity, FEBS letters 579, 705-711.

[94] Kao, T.-K., Ou, Y.-C., Raung, S.-L., Lai, C.-Y., Liao, S.-L., and Chen, C.-J. (2010) Inhibition of nitric oxide production by quercetin in endotoxin/cytokine-stimulated microglia, Life sciences 86, 315-321.

[95] Wang, K., Ping, S., Huang, S., Hu, L., Xuan, H., Zhang, C., and $\mathrm{Hu}, \mathrm{F}$. (2013) Molecular mechanisms underlying the in vitro antiinflammatory effects of a flavonoid-rich ethanol extract from Chinese propolis (poplar type), Evidence-Based Complementary and Alternative Medicine 2013.
[96] Amic, D., Davidovic-Amic, D., Beslo, D., Rastija, V., Lucic, B., and Trinajstic, N. (2007) SAR and QSAR of the antioxidant activity of flavonoids, Current medicinal chemistry 14, 827-845.

[97] Zhang, J., Cao, X., Ping, S., Wang, K., Shi, J., Zhang, C., Zheng, H., and Hu, F. (2015) Comparisons of ethanol extracts of Chinese propolis (poplar type) and poplar gums based on the antioxidant activities and molecular mechanism, Evidence-Based Complementary and Alternative Medicine 2015.

[98] Nakanishi, I., Uto, Y., Ohkubo, K., Miyazaki, K., Yakumaru, H., Urano, S., Okuda, H., Ueda, J.-I., Ozawa, T., and Fukuhara, K. (2003) Efficient radical scavenging ability of artepillin C, a major component of Brazilian propolis, and the mechanism, Organic \& biomolecular chemistry 1, 1452-1454.

[99] Lotito, S. B., and Frei, B. (2006) Dietary flavonoids attenuate tumor necrosis factor $\alpha$-induced adhesion molecule expression in human aortic endothelial cells structure-function relationships and activity after first pass metabolism, Journal of Biological Chemistry 281, 37102-37110.

[100] Wu, W.-M., Lu, L., Long, Y., Wang, T., Liu, L., Chen, Q., and Wang, R. (2007) Free radical scavenging and antioxidative activities of caffeic acid phenethyl ester (CAPE) and its related compounds in solution and membranes: a structure-activity insight, Food Chemistry 105, 107-115. 